Meta

Journal des traducteurs

Translators' Journal

\title{
Le traducteur professionnel face aux textes techniques et à la recherche documentaire
}

\section{Laurent Lagarde et Daniel Gile}

Volume 56, numéro 1, mars 2011

URI : https://id.erudit.org/iderudit/1003517ar

DOI : https://doi.org/10.7202/1003517ar

Aller au sommaire du numéro

Éditeur(s)

Les Presses de l’Université de Montréal

ISSN

0026-0452 (imprimé)

1492-1421 (numérique)

Découvrir la revue

Citer cet article

Lagarde, L. \& Gile, D. (2011). Le traducteur professionnel face aux textes techniques et à la recherche documentaire. Meta, 56(1), 188-199.

https://doi.org/10.7202/1003517ar

\section{Résumé de l'article}

Le présent article rend compte des comportements de recherche documentaire chez des traducteurs techniques indépendants sur la base d'une analyse alternant questionnaires et entretiens avec mise au point progressive. Il s'agissait notamment d'examiner ces comportements au regard de paramètres tels que la spécialisation, la formation préalable, l'expérience et les langues de travail des répondants. Les résultats, obtenus sur un ensemble de 245 répondants, donnent à penser que tous ces paramètres influent effectivement sur les comportements. La pression du temps et Internet se sont également avérés des déterminants importants. Enfin, les réponses obtenues montrent que la pratique sur le terrain est souvent éloignée des principes enseignés en formation. 


\title{
ÉTUDES ET PROSPECTIVES
}

\section{Le traducteur professionnel face aux textes techniques et à la recherche documentaire}

\author{
LAURENT LAGARDE* \\ Lyon, France \\ laurentlagarde@hotmail.com \\ DANIEL GILE \\ ESIT - Université Sorbonne Nouvelle - Paris 3, Paris, France \\ daniel.gile@yahoo.com
}

\begin{abstract}
RÉSUMÉ
Le présent article rend compte des comportements de recherche documentaire chez des traducteurs techniques indépendants sur la base d'une analyse alternant questionnaires et entretiens avec mise au point progressive. Il s'agissait notamment d'examiner ces comportements au regard de paramètres tels que la spécialisation, la formation préalable, l'expérience et les langues de travail des répondants. Les résultats, obtenus sur un ensemble de 245 répondants, donnent à penser que tous ces paramètres influent effectivement sur les comportements. La pression du temps et Internet se sont également avérés des déterminants importants. Enfin, les réponses obtenues montrent que la pratique sur le terrain est souvent éloignée des principes enseignés en formation.
\end{abstract}

\section{ABSTRACT}

This paper reports on information acquisition patterns ("research") in freelance technical translators based on a series of questionnaires alternating with interviews, and fine-tuned along the way. The principal aim was to identify links between these patterns and other parameters such as translators' previous specialization, training, experience and working languages. The analysis of responses by 245 translators suggests that all these parameters do indeed have an effect on information acquisition patterns and that time pressure as well as the Internet are also important determinants of such behavior. Findings also show that actual field practice is often quite remote from principles taught during training.

\section{MOTS-CLÉS/KEYWORDS}

traduction technique, acquisition de connaissances, termes spécialisés, pression du temps, stratégies, méthodologie d'alternance

technical translation, knowledge acquisition, specialized terms, time pressure, strategies, alternation methodology

\section{Introduction}

Aujourd'hui, la traduction de textes non littéraires domine le marché de la traduction professionnelle, en raison notamment de l'importance des sciences et des techniques dans le monde. Différents travaux portent sur le profil des traducteurs indépendants (Cancio-Pastor 1995), sur les relations entre le traducteur et son client (Fraser 1999), 
sur les points forts et les faiblesses des formations de traducteurs (Bouderradji 2004), ainsi que sur les tactiques (souvent appelées «stratégies») des apprentis traducteurs et des professionnels (Krings 1986; Jääskeläinen 1987; Jääskeläinen 1989; TirkkonenCondit 1989; Dancette 1995; Lörscher 1996; Königs et Kaufman 1996; Künzli 2003; Hébert-Malloch 2004; parmi beaucoup d'autres). La recherche documentaire est analysée par divers auteurs (voir notamment Durieux 1988; Gouadec 2002; Gile 2005; Plassard 2007a), mais comme le notait déjà Künzli en 2001, les travaux empiriques consacrés aux stratégies et tactiques effectivement mises en œuvre sur le terrain sont peu nombreux. Pour la plupart, ils sont expérimentaux et impliquent une manipulation ou un contrôle sur le processus et/ou sur l'environnement du traducteur et font appel à des méthodes introspectives, par rétrospection (après la traduction) ou «en ligne» (pendant la traduction) (voir par exemple Künzli 2001 ou Göpferich 2008). Ils suscitent d'ailleurs des interrogations sur la validité écologique des méthodes employées (voir par exemple une discussion de la validité des protocoles de verbalisation à voix haute dans Englund Dimitrova 2005 : 70-75). Dès lors, il apparaît intéressant de chercher à compléter et recouper leurs résultats avec des méthodes naturalistes (que nous définissons ici comme des méthodes analysant les phénomènes tels qu'ils se déroulent «naturellement», sans manipulation de la tâche ou de l'environnement).

Dans cet esprit, pour enrichir et affiner les connaissances fragmentaires dont on dispose actuellement et qui proviennent de l'observation individuelle sur le terrain et de l'examen systématique des comportements des participants lors d'études expérimentales, des informations ont été recueillies par questionnaires et interviews. L'effort a essentiellement porté sur la recherche de l'influence potentielle d'un certain nombre de paramètres peu pris en compte jusqu'à présent (notamment la formation du traducteur, son expérience et ses langues de travail) sur les attitudes et la pratique en matière d'acquisition de connaissances ad hoc aux fins de la traduction.

\section{L'acquisition de connaissances ad hoc dans la traduction}

\subsection{Le modèle séquentiel de la traduction et l'acquisition de connaissances ad hoc}

Pour analyser les tactiques de traduction et les stratégies de recherche documentaire, c'est le cadre conceptuel du modèle séquentiel de la traduction (Gile 2005) qui a été choisi. Ce modèle met en exergue l'importance des connaissances du traducteur dans le processus de traduction; il représente celui-ci en deux étapes récursives, la phase de compréhension et la phase de reformulation (Figure 1).

Dans la première étape, le traducteur lit une unité de traduction, en extrait une hypothèse de sens puis en vérifie la plausibilité, en construit éventuellement une deuxième et en vérifie la plausibilité et ainsi de suite jusqu'à adoption de l'une des hypothèses. Dans la deuxième phase, sur la base de l'hypothèse de sens retenue, il rédige un énoncé d'arrivée, puis en vérifie la fidélité au sens de l'original et l'acceptabilité rédactionnelle, le modifie le cas échéant, et ainsi de suite jusqu'à l'adoption d'un énoncé définitif (Gile 2005: 103). Ses connaissances existantes (sa «base de connaissances») peuvent être mobilisées à toutes les étapes du processus, mais elles doivent régulièrement être complétées par l'acquisition de connaissances ad hoc, processus qui est au centre de l'étude présentée ici. 


\section{FIGURE 1}

Modèle séquentiel de la traduction (Gile 2005: 102)

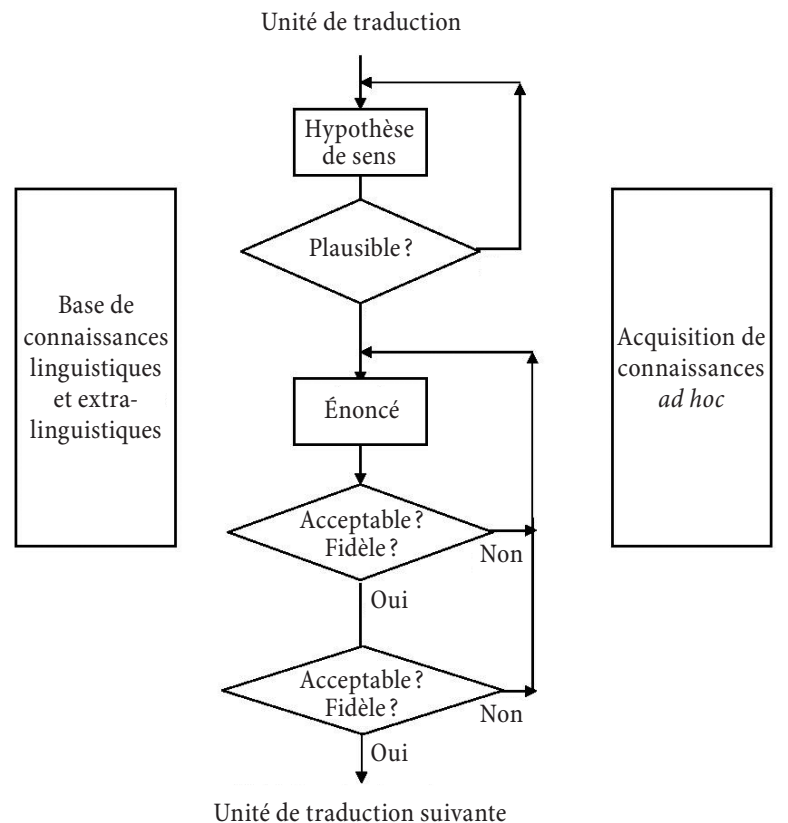

\subsection{Sources pour l'acquisition de connaissances ad hoc}

Aux fins de l'analyse, nous adoptons ici la taxonomie proposée par Gile (2005):

\subsubsection{Sources documentaires}

Il s'agit de textes, sur support papier ou électroniques (notamment sur Internet). Ils peuvent être «terminologiques» (glossaires, dictionnaires, bases de données terminologiques...) ou «non terminologiques» (textes de fond, autres textes divers).

\subsubsection{Sources humaines}

Il s'agit de personnes à qui le traducteur fait appel pour l'aider à compléter des connaissances manquantes. Selon le cas, ce sont des documentalistes ou terminologues travaillant au sein de l'organisation dont le traducteur est salarié, des spécialistes du sujet dont traite le texte à traduire qu'il consulte à titre ponctuel ou régulier, des collègues traducteurs qu'il consulte à titre individuel ou dans le cadre d'un réseau formel de traducteurs (Plassard 2007b).

\section{Méthodologie}

Les traducteurs professionnels connaissent bien leur propre pratique en matière d'acquisition de connaissances ad hoc ainsi que celle des collègues qu'ils fréquentent régulièrement; cependant, cette connaissance est par définition fragmentaire: on ne 
dispose pas à l'heure actuelle d'informations sur le tableau d'ensemble et sur l'influence de différents facteurs «environnementaux» sur ces comportements. L'étude présentée ici visait à poser des jalons dans l'exploration de cette réalité professionnelle. Réalisée entre 2003 et 2006, elle a consisté à recueillir des informations écrites et orales à l'aide de questionnaires et d'entretiens individuels.

\subsection{L'échantillon}

L'étude s'est focalisée sur les pratiques des traducteurs techniques indépendants, par opposition aux salariés, dont la situation au regard de l'environnement de travail et des besoins et pratiques d'acquisition de connaissances ad hoc est assez différente.

La constitution par les méthodes statistiques classiques d'un échantillon représentatif de l'ensemble des traducteurs indépendants et des différentes valeurs des paramètres concernés (formation, langues de travail, domaines de spécialité) ne semblait pas être une stratégie très efficace, avec un risque élevé de «cellules» vides ou trop peu peuplées faute de pouvoir accéder à des répondants en nombre suffisant. Pour une première étude exploratoire, la stratégie d'échantillonnage la plus prometteuse a consisté plutôt à viser un nombre de témoignages aussi grand que possible, sachant que, de ce fait, la représentativité de l'échantillon à l'égard des différents paramètres n'est pas assurée et que toute généralisation est nécessairement risquée. Des études similaires sur des échantillons représentatifs de sous-populations particulières de traducteurs permettront ultérieurement de vérifier et d’affiner nos résultats.

Les traducteurs sollicités étaient connus de l'auteur principal du présent article ou figuraient dans des annuaires téléphoniques et des sites Internet, ou encore, ils se sont montrés accessibles lors de rencontres professionnelles. La majorité des 245 traducteurs professionnels qui ont accepté de participer travaillaient en France (213 sur 245 , soit $86,9 \%)$, les autres $(13,1 \%)$ exerçaient dans quinze autres pays ${ }^{1}$. Les répondants résidant en France ont été contactés initialement par téléphone. Ce premier contact, qui a permis de leur présenter l'étude, s'est révélé efficace, puisqu'un grand nombre d'entre eux ont accepté de répondre aux questions. Pour des raisons pratiques, les traducteurs exerçant à l'étranger ont été contactés uniquement par courriel; cette méthode a eu moins de succès que la prise de contact par téléphone, bon nombre de traducteurs n'ayant pas répondu. Sur les 188 questionnaires envoyés à des traducteurs exerçant en France, 171 (91\%) ont été retournés. Le taux de réponse a été de 30,7\% pour les traducteurs résidant à l'étranger (20 questionnaires retournés sur 65).

L'échantillon couvre 29 langues de travail ${ }^{2}$, le français (92,3\% des répondants), l'anglais (42,1\%) et l'allemand (17,1\%) étant les langues plus représentées. Vingt-deux des vingt-neuf langues recensées $(75,8 \%)$ étaient peu répandues dans le pays où travaillaient les répondants, mais elles ne concernaient que 52 traducteurs sur 245 , soit $21,2 \%$.

Deux cent six répondants $(84,1 \%)$ avaient au moins un domaine de spécialité: le droit, l'informatique, l'économie, les transports, les disciplines biomédicales et la pharmacologie constituent $60,9 \%$ des domaines de spécialité évoqués.

La grande majorité des répondants $(75,5 \%)$ étaient diplômés d'une école de traduction ou avaient une formation universitaire en traduction professionnelle.

Enfin, les répondants avaient quinze ans d'expérience en moyenne (fourchette: 1 à 35 ans). 


\subsection{Entretiens et questionnaires}

Les informations recueillies portaient sur les tactiques de traduction et sur les stratégies de recherche documentaire. Deux outils d'investigation complémentaires ont été mis en œuvre: les entretiens et les questionnaires.

\subsubsection{Les entretiens}

Il s'agit d'une méthode plutôt ouverte, qui permet, dans le cadre d'un dialogue, d'obtenir de nombreuses informations. Les entretiens se prêtent aux analyses qualitatives plus qu'aux analyses quantitatives. Celles-ci ne sont toutefois pas exclues, dans la mesure où l'on peut tirer lors d'un entretien des réponses précises à certaines questions et les comptabiliser par la suite.

En revanche, organiser des entretiens est parfois difficile et demande un temps considérable; la plupart des traducteurs contactés n'ont pu se libérer pour un entretien que plusieurs semaines après le contact initial. Par ailleurs, l'échantillon de répondants interviewés était relativement restreint du fait de contraintes géographiques liées à la nécessité de rencontrer chacun face à face.

\subsubsection{Les questionnaires}

Ceux-ci ont permis d'augmenter le nombre de répondants et de réaliser davantage d'analyses quantitatives. Ils ont toutefois été moins productifs, les réponses données étant souvent brèves et ne permettaient pas un approfondissement par questions complémentaires immédiates.

\subsection{La progression par alternance}

Plutôt que de réaliser l'opération en plusieurs étapes (y compris des étapes pilotes pour la mise au point des outils), étant donné les contraintes et les limites propres à chacune de ces méthodes ainsi que le nombre peu élevé de traducteurs accessibles, nous avons eu recours à une alternance de questionnaires et d'entretiens. Ceci a permis de maximiser l'efficacité de la procédure: ainsi, les informations recueillies grâce aux questionnaires pouvaient être exploitées et, en même temps, servir à la préparation des entretiens qui les suivaient; ces entretiens permettaient ensuite d'affiner les questionnaires ultérieurs, et ainsi de suite.

Cette stratégie a permis d'exploiter un maximum d'entretiens et de questionnaires, de mettre au point les outils au fil de la progression et de les cibler sur des éléments qui semblaient mériter approfondissement. Elle aboutit cependant à des tailles d'échantillons qui ne sont pas les mêmes pour chacune des questions abordées, comme le montre plus loin la présentation des résultats.

Les questionnaires thématiques (d'approfondissement) portaient sur l'identification des problèmes dans le texte de départ à différents moments, sur la démarche adoptée dans l'acquisition de connaissances ad hoc en fonction de la technicité du texte de départ, sur l'utilisation d'Internet, sur les problèmes posés par la compréhension des sources documentaires, sur la consultation des sources humaines, sur la traduction des termes techniques empruntés au vocabulaire courant, sur les stratégies d'archivage et sur les problèmes spécifiques se posant à propos des langues peu répandues (LPR) définies comme celles qui, dans le pays de résidence du traducteur, sont peu utilisées et ont une importance économique faible. 
Au total, le corpus analysé est constitué par les réponses obtenues lors de trois séries d'entretiens individuels organisés avec 54 traducteurs et trois séries de questionnaires remplis par 191 traducteurs.

\section{Synthèse des résultats}

\subsection{Le facteur temps}

Premier résultat frappant: la pression exercée par le temps semble avoir une importance considérable sur le travail et les décisions du traducteur, souvent dans un sens qui va à l'encontre des principes et conseils formulés dans les programmes de formation.

Ainsi, quand les délais sont courts, la lecture du texte de départ n'est pas systématique, surtout s'il est long et si le traducteur connaît bien le sujet (62 personnes sur 62 interrogées sur cette question).

Par ailleurs, quand le temps est compté, nos répondants préfèrent en majorité utiliser des sources terminologiques plutôt que des sources non terminologiques (43 personnes sur 65 qui ont répondu à la question, soit 66,1\% des répondants). Ils en connaissent les limites, mais elles sont plus rapides d'utilisation que les sources non terminologiques et leur donnent des réponses immédiates. Une majorité de répondants considèrent d'ailleurs que se rendre dans une bibliothèque pour consulter des sources documentaires est une perte de temps, car ils trouveront toujours des sources en ligne répondant à leurs besoins (59 personnes sur 74 interrogées, soit $79,7 \%$ ). Cette modification des comportements liée à Internet n'est que l'une des conséquences de l'arrivée de la Toile dans l'environnement du traducteur (voir plus loin).

Les contraintes temporelles semblent aussi limiter, dans une grande mesure, la démarche d'approfondissement général des connaissances chez les traducteurs; $84 \%$ des répondants (42 sur 50) ne prennent pas le temps de faire des recherches au-delà des termes spécialisés qu'ils rencontrent au fil des traductions qu'ils effectuent. Plus généralement, la majorité des répondants disent ne pas avoir le temps d'acquérir hors traduction des connaissances complémentaires dans leurs domaines de spécialité (81 sur 97, soit 83,5\%). Ces réponses corroborent l'observation de Mareschal, pour qui l'acquisition de connaissances chez le traducteur tend à se limiter à « une recherche ponctuelle et non thématique, [...] portant sur des termes isolés ou sur des groupes restreints de termes d'un même domaine [...]» (Mareschal 1989: 259).

De même, la pression du temps les empêche de faire le point sur les ouvrages dont ils ont besoin pour se spécialiser et acquérir des connaissances complémentaires (pour 68 répondants sur 84, soit 80,9\%), ou d'archiver les informations terminologiques et phraséologiques qu'ils recueillent (59 traducteurs sur 78, soit 75,6\%).

La pression du temps influe également, de manière un peu inattendue, sur l'utilisation des mémoires de traduction. En effet, $71 \%$ des répondants (51 sur 72 ) n'utilisent pas ces outils; une majorité d'entre eux dit ne pas avoir le temps d'apprendre à en maîtriser toutes les fonctionnalités (35 répondants sur 55, soit 63,6\%). Une étude, menée par Arrouart (2003), a dressé un premier constat de l'influence des mémoires de traduction sur la pratique traduisante et a notamment montré que le temps passé à apprendre à utiliser ces outils est important, qu'ils sont onéreux et que les donneurs d'ouvrage révisent souvent leurs tarifs à la baisse quand le traducteur les utilise. 


\subsection{Les langues de travail}

Les langues de travail s'avèrent avoir une grande influence sur l'activité et la manière de travailler du traducteur. Des différences importantes apparaissent à cet égard entre les traducteurs de langues répandues (LR) et les traducteurs de langues peu répandues (LPR) dans leur pays de résidence.

Sur le plan économique, les traducteurs de LPR ont un potentiel de clients moins important que les traducteurs de LR, si bien qu'en dehors des traducteurs assermentés, ils ne se cantonnent que rarement dans des domaines de spécialité précis sous peine de restreindre leur marché. Pour la même raison, ils sont relativement nombreux à traduire dans les deux sens (de et vers la LPR) et certains proposent à leurs clients une combinaison de langues supplémentaire, par exemple la combinaison anglais-français (20 sur 52, soit 38,4\%).

Par ailleurs, la majorité des traducteurs de LPR interrogés font état de problèmes de documentation, les sources à leur disposition dans cette langue étant apparemment moins nombreuses, moins complètes et moins fiables que les sources en LR (40 sur 52 , soit $76,9 \%)$. Internet a quelque peu amélioré la situation; la plupart des traducteurs de langues nordiques considèrent que les sources en ligne disponibles dans ces langues offrent aujourd'hui une bonne complétude (10 sur 14, soit 71,4\%), ce qui n'est pas encore le cas des documents en ligne que trouvent les traducteurs de langues d'Asie et d'Europe de l'Est (16 sur 18, soit 88,8\%). En raison du manque de sources Internet, les documents sur support papier occupent toujours une place centrale dans la recherche documentaire chez les traducteurs de LPR. Près de la moitié d'entre eux utilisent Internet après avoir consulté des documents sur support papier (23 sur 48, soit $47,9 \%)$.

Le manque de sources implique des stratégies de recherche documentaire et des tactiques de traduction que l'on ne trouve pas souvent chez les traducteurs de LR. Ainsi, tous les traducteurs de LPR (35 sur 35) utilisent des sources rédigées dans une langue tierce et une partie d'entre eux (15 sur 35, soit 42,8\%) consultent davantage de documents écrits dans la LR que dans la LPR. En outre, près de $91 \%$ des traducteurs de LPR (30 sur 33) ont expliqué que le manque de documents impliquait un effort de création terminologique de leur part. Si ce phénomène était confirmé par d'autres études, il serait intéressant d'en étudier les incidences éventuelles sur les langues de spécialité concernées : l'influence des traducteurs y est-elle grande? Si c'est le cas, doit-on s'interroger sur l'opportunité de leur offrir une formation à la terminologie, afin que les néologismes proposés soient bons? C'est en tout cas ce que réclament certains en Grèce, où la problématique est un peu différente et les besoins portent essentiellement sur la traduction de termes anglais vers le grec (Galani 2010).

Par ailleurs, plus de la moitié des traducteurs de LPR de notre échantillon font appel aux spécialistes plus systématiquement que les traducteurs de LR (31 sur 52, soit $59,6 \%$ ). Ils sont toutefois conscients du fait que cette démarche prend du temps et qu'il n'existe pas de spécialistes connaissant la LPR et capables de leur apporter une aide efficace pour tous les sujets. L'archivage des informations terminologiques et phraséologiques semble être plus systématique chez les traducteurs de LPR que chez les traducteurs de LR; le manque de documents pousse la majorité d'entre eux à garder précieusement les informations trouvées (39 sur 47, soit 82,9\%). Les stratégies d'achat de documents sont également différentes; si les traducteurs de LR achètent 
de moins en moins de sources sur support papier, les traducteurs de LPR (28 sur 39, soit 71,9\%) s'efforcent d'en acheter un grand nombre (notamment des sources terminologiques de tous domaines).

\subsection{Généralistes et spécialistes}

Les pratiques des généralistes et des traducteurs spécialisés diffèrent sur de nombreux aspects.

Une première différence, légère, se manifeste dans l'importance qu'ils accordent à la technicité du texte de départ. Avant d'accepter de le traduire, les généralistes (33 sur 45 , soit $73,3 \%$ ) accordent plus d'importance à sa technicité que les traducteurs spécialisés (25 sur 36, soit 69,4\%).

Par ailleurs, une majorité de répondants spécialisés dans des domaines où l'évolution technique et scientifique est rapide, tels que la médecine et l'informatique, disent utiliser davantage Internet parce que les documents sur support papier sont rapidement dépassés (52 répondants sur 69, soit 75,3\%), alors que la majorité des traducteurs spécialisés dans des domaines à faible évolution technique et scientifique, tels que le droit, consultent en priorité des sources sur papier, même s'ils en possèdent peu (26 sur 42 , soit $61,9 \%)$.

Les stratégies d'achat des généralistes s'apparentent à celles des traducteurs de LPR; ils évitent de focaliser leurs achats sur un domaine précis et achètent en priorité des dictionnaires de tous domaines techniques, car ils ont des besoins ponctuels variés $\left(71,4 \%\right.$ des traducteurs généralistes de $\mathrm{LR}^{3}$ et 71,8\% des traducteurs de $\left.\mathrm{LPR}^{4}\right)$.

\subsection{Formation de spécialiste et expérience}

La formation éventuelle du traducteur dans un domaine de spécialité et l'expérience de la traduction professionnelle paraissent avoir elles aussi une influence sur la pratique de la traduction. La plupart des répondants qui ont un niveau élevé de connaissances dans un domaine de spécialité utilisent en priorité des sources non terminologiques très techniques ( 9 sur 12, soit $75 \%$ ) alors que ceux qui ont peu de connaissances spécialisées utilisent des documents moyennement techniques (9 sur 15 , soit $60 \%)$.

Par ailleurs, les traducteurs expérimentés n'ont pas la même perception du texte technique et de son niveau de difficulté que les «jeunes » traducteurs. Les répondants qui voient un lien fort entre la technicité du texte et sa «difficulté» ont moins d'expérience que ceux qui n'en voient pas (10,5 ans d'expérience en moyenne contre 16,5 respectivement). Les «jeunes» traducteurs se focalisent davantage que les traducteurs expérimentés sur les termes d'apparence très technique qu'ils repèrent lors de la première lecture du texte de départ; les traducteurs expérimentés ont davantage tendance à considérer qu'un texte contenant un grand nombre de termes de cette catégorie n'est pas forcément plus difficile à traduire et qu'il n'y a pas forcément une forte corrélation entre la technicité du texte et la difficulté de sa traduction (20,3 ans d'expérience en moyenne contre 11,3 pour ceux qui pensent le contraire).

En revanche, de manière plus générale, les traducteurs expérimentés ont plus de réticences que les «jeunes traducteurs» à accepter des textes très techniques (15,8 années d'expérience en moyenne pour les premiers contre 11 ans pour les 
seconds); les traducteurs expérimentés sont moins enclins à accepter un texte sur un sujet qu'ils connaissent mal et considèrent qu'Internet n'est pas la solution qui va leur permettre d'acquérir toutes les connaissances dont ils ont besoin pour traduire un texte. Les traducteurs expérimentés passent plus de temps à analyser les sources non terminologiques qu'ils utilisent et sont plus à même de comprendre le sociolecte spécialisé alors que les «jeunes» traducteurs préfèrent exploiter des sources terminologiques qui donnent des solutions immédiates à des difficultés de traduction (15,3 ans d'expérience en moyenne pour les premiers contre 9,5 pour les seconds) ${ }^{5}$.

Les «jeunes» traducteurs ont plus de difficulté à évaluer l'utilité et la fiabilité d'une source documentaire que leurs collègues expérimentés (12 ans d'expérience en moyenne contre 20,3 respectivement) ${ }^{6}$. Enfin, ils considèrent qu'il est plus facile de se spécialiser en utilisant des sources en ligne qu'avec des documents sur support papier (11 ans d'expérience contre 16,4 ans pour ceux qui pensent le contraire).

\subsection{Internet}

Comme le montrent déjà les réponses ci-dessus, l'émergence d'Internet semble avoir eu des répercussions considérables sur les supports utilisés et sur les stratégies de recherche documentaire. Aujourd'hui, les traducteurs consultent davantage de sources en ligne que de documents sur support papier (34 sur 44 répondants, soit 77,2 \%). Pour la majorité des répondants, Internet offre un grand confort d'utilisation et un nombre considérable d'informations (50 sur 75 personnes, soit 66,6\%); les avantages de cet outil ont marginalisé les sources sur support papier, même si les documents en ligne ne donnent pas toujours de réponses directes à leurs requêtes. Les trois quarts des répondants qui se sont prononcés sur ce sujet (63 sur 84 ) préfèrent les documents en ligne. Comme le suggéraient déjà des réponses résumées plus haut, la recherche sur place, en bibliothèque ou dans d'autres établissements comportant des documents «papier», est en voie de disparition; 79,7\% des répondants (59 sur 74) disent ne plus pratiquer cette forme de recherche documentaire.

Et pourtant, cette évolution ne semble pas avoir accru l'isolement des traducteurs. Peut-être sont-ils même davantage en contact avec le monde extérieur qu'auparavant, car de véritables communautés virtuelles de traducteurs se sont constituées avec la création de sites comme Proz ${ }^{7}$ ou Translatorscafe ${ }^{8}$; il y est possible de poser des questions terminologiques et phraséologiques et de trouver des offres de traduction déposées par des clients (Gambier 2007; Mc Donough 2007; Plassard 2007b). La qualité des informations terminologiques et phraséologiques échangées sur ces sites reste à étudier. Notons à ce propos que Proz et Translatorscafe ont un système d'évaluation des réponses données; le traducteur pose une question terminologique ou phraséologique et évalue les réponses données par les utilisateurs du site.

Si Internet offre des avantages incontestables, il ne permet pas forcément de se spécialiser; plus de la moitié des répondants ( 84 sur 149, soit 56,3\%) considèrent qu'il est souvent difficile de trouver des sources qui reprennent les connaissances à la base d'un domaine précis. Les deux tiers des traducteurs qui se sont prononcés sur ce point (50 sur 75) gardent une certaine méfiance envers les documents en ligne, leur fiabilité étant incertaine du fait que beaucoup de non-spécialistes écrivent sur Internet; ils considèrent que les auteurs de documents sur support papier ont plus d'autorité. L'émergence d'Internet aurait également influencé les délais accordés par les clients 
qui, d'après $77,7 \%$ des répondants (77 sur 99$)$, raccourciraient ces délais sous prétexte que le traducteur n'aurait plus de problème d'accès à l'information. Enfin, la majorité des répondants ( 72 sur 108, soit 66,6\%) disent accepter de traduire des textes plus techniques qu'avant l'existence d'Internet, car ils peuvent maintenant trouver en ligne des sources très spécialisées.

\section{Discussion méthodologique}

Au cours de cette étude, un certain nombre de questions et de défis méthodologiques sont apparus comme particulièrement saillants.

Premièrement, lors des entretiens, les répondants ne comprenaient pas toujours pourquoi ils étaient questionnés sur des aspects précis de leur pratique auxquels ils n'avaient pas nécessairement réfléchi. Ils affirmaient qu'il était impossible de schématiser leur travail comme semblait le faire le chercheur, que les questions posées ne s'inscrivaient pas dans leur réalité quotidienne. Il a donc fallu fournir des explications qui ont peut-être influencé les réponses obtenues par la suite.

Deuxièmement, certaines réponses demandaient confirmation, approfondissement, ou les deux, ce qui a rendu les entretiens et les questionnaires de plus en plus longs, au point que leur longueur devînt parfois problématique. Par manque de temps, certaines questions n'ont pu être abordées pendant les entretiens, mais des questionnaires trop longs ne pouvaient que réduire les chances d'obtenir des réponses complètes. Il a donc parfois été nécessaire d'arbitrer et de privilégier les questions demandant un approfondissement sur celles nécessitant une confirmation.

Troisièmement, les questionnaires ne sont pas la méthode d'investigation la plus efficace pour aborder certains aspects de la traduction qui demandent de la part du répondant un vrai travail d'introspection. Les entretiens ont pu compenser la rigidité des questionnaires, car ils ont été l'occasion de reformuler des questions et de demander des précisions quand les réponses n'étaient pas pertinentes, donc à orienter les répondants vers une réflexion introspective.

Bien entendu, la question de la fiabilité des informations recueillies est essentielle. Les réponses des traducteurs parlant de leur pratique quotidienne à un collègue traducteur comportaient nécessairement une part de subjectivité et n'étaient peutêtre pas exemptes d'éléments valorisants ou défensifs, par exemple dans les propos de certains répondants affirmant que, malgré la pression du temps, ils s'efforçaient d'acquérir des connaissances sur des questions dépassant les problèmes précis rencontrés dans les textes qu'ils traduisaient, ou qu'ils archivaient systématiquement les termes et les tournures phraséologiques rencontrés dans ces textes ou trouvés dans des sources extérieures. Pour réduire un tant soit peu l'impact de ces phénomènes bien connus dans le monde de la recherche par enquêtes, l'intervieweur a insisté sur les contraintes et difficultés du métier afin de mettre à l'aise ses interlocuteurs et les aider à donner des réponses aussi proches que possible de la réalité, mais il est difficile d'évaluer l'effet de cette stratégie.

De ce fait, la présente étude ne saurait être autre chose qu'une exploration partielle, dont les résultats doivent être confirmés et complétés avec d'autres méthodes comportant l'observation effective des comportements. 


\section{Conclusion}

Cette étude, réalisée sur un échantillon de traducteurs assez important, confirme une réalité qui ne surprendra guère les professionnels: les pratiques sont parfois assez éloignées de l'idéal enseigné, et les contraintes de temps y jouent un rôle déterminant.

Il serait peu réaliste d'espérer s'affranchir de la pression des délais trop courts sur le marché ou d'une partie de ses effets néfastes, notamment la nécessité de livrer au client des traductions sans avoir le temps de prendre suffisamment de recul avant de s'auto-réviser.

Une réponse partielle au problème pourrait se situer dans une meilleure utilisation d'Internet, qui s'enrichit à grande vitesse d'une masse de documents - dont la fiabilité est toutefois sujette à caution dans de nombreux cas. Des études exploratoires consacrées à cette ressource et à son utilisation au quotidien par les traducteurs ne seraient pas dénuées d'intérêt, de même que des formations spécifiques à son exploitation. Une utilisation plus systématique, et peut-être professionnelle, voire commerciale, des réseaux sociaux de traducteurs, ou alors une coopération institutionnalisée avec des organismes techniques dans différents domaines de spécialité aboutissant à des systèmes de certification de qualité de documents en ligne, devraient permettre elles aussi de progresser vers une meilleure qualité générale de l'acquisition d'informations ad hoc dans la traduction spécialisée.

\section{NOTES}

* Laurent Lagarde est traducteur indépendant. Le présent article est fondé sur les résultats obtenus dans le cadre de son travail doctoral effectué sous la direction de Daniel Gile (Lagarde 2009).

1. Allemagne, Argentine, Brésil, Danemark, Espagne, États-Unis, Finlande, Grèce, Israël, Italie, Liban, Nouvelle-Zélande, Portugal, Royaume-Uni et Suède.

2. Anglais, arabe, basque, bulgare, catalan, chinois, coréen, danois, espagnol, finnois, français, grec, hébreu, hongrois, italien, japonais, néerlandais, norvégien, persan, polonais, portugais, roumain, russe, slovaque, suédois, tchèque, turc, ukrainien et yiddish.

3. 50 sur 70

4. 28 sur 39 .

5. Chiffres obtenus en comparant l'expérience des traducteurs avec les réponses à la question de l'analyse des sources non terminologiques.

6. Chiffres obtenus à partir des informations données sur leur expérience et des réponses données sur la question de l'évaluation de l'utilité et de la fiabilité des sources documentaires.

7. <www.proz.com>, consultée le 22 juin 2010.

8. <www.translatorscafe.com>, consultée le 22 juin 2010.

\section{RÉFÉRENCES}

Arrouart, Catherine (2003): Les mémoires de traduction et la formation universitaire: quelques pistes de réflexion. Meta. 48(3):476-479.

BOUDERRADJI, Khadija (2004): Formation des traducteurs et réalité professionnelle: harmonie ou discordance? Mémoire de DEA non publié. Lyon: Université Lyon II Lumière.

CAnCIO-PAstor, Carmelo (1995): La traduction professionnelle en France: approche du domaine hispanique. Thèse de doctorat non publiée. Toulouse: Université Toulouse II Le Mirail.

Dancette, Jeanne (1995): Parcours de traduction: étude expérimentale du processus de compréhension. Lille: Presses universitaires de Lille.

Durieux, Christine (1988): Fondements didactiques de la traduction technique. Paris: Didier Érudition.

Englund Dimitrova, Birgitta (2005): Expertise and Explicitation in the Translation Process. Amsterdam/Philadelphia: John Benjamins. 
Fraser, Janet (1999): L'apprentissage de la vie quotidienne des traducteurs spécialisés. In: Daniel GouAdEC, dir. Formation des traducteurs. (Formation des traducteurs - Colloque international de l'Université Rennes II, Rennes, 24-25 septembre 1999). Paris: La maison du dictionnaire, 83-87.

Galani, Dimitra (2010): Traduction et terminologie: une étude de cas sur la problématique terminologique en Grèce. Mémoire de Master II non publiée. Paris: ESIT - Université Sorbonne Nouvelle-Paris 3.

GAmbier, Yves (2007): Réseaux de traducteurs/interprètes bénévoles. Meta. 52(4):658-672.

GiLE, Daniel (2005): La traduction: la comprendre, l'apprendre. Paris: Presses universitaires de France.

GöPferich, Susanne (2008): Translationsprozessforschung. Stand, Methoden, Perspektiven. Tübingen: Gunter Narr.

Gouadec, Daniel (2002): Profession: traducteur. Paris: Maison du dictionnaire.

Hébert-Malloch, Louise (2004): What do we know about a translator's day? Meta. 49(4):973979.

JäÄSKELÄINEN, Riitta (1987): What happens in a translation process: think-aloud protocols of translation. Unpublished pro graduation thesis. Joensuu: Savonlinna School of Translation Studies - University of Joensuu.

JäÄSKELÄInEN, Riitta (1989): The role of reference material in professional vs. non-professional translation: A think-aloud protocol study. In: Sonia Tirkkonen-Condit et Stephen Condit, dir. Empirical Studies in Translation and Linguistics. Joensuu: University of Joensuu, 175-200.

Königs, Frank et Kaufmann, Rheinard (1996): Processus mentaux étudiés chez des sujets allemands apprenant le français lorsqu'ils sont en train de traduire. Résultats d'une petite étude de cas comme constitution à la didactique de la traduction et de l'enseignement des langues étrangères. Meta. 41(1):7-26.

KRINGS, Hans Peter (1986): Was in den Köpfen von Übersetzern vorgeht, eine empirische Untersuchung zur Struktur des Übersetzungsprozesses an fortgeschrittenen Französischlernern. Tübingen: Gunter Narr.

KüNZLI, Alexander (2001) : Experts versus novices: l'utilisation de sources d'information pendant le processus de traduction. Meta. 46(3):507-523.

KüNZLI, Alexander (2003): Quelques stratégies et principes en traduction technique françaisallemand et français-suédois. Thèse de doctorat publiée. Stockholm: Université de Stockholm.

LAGARDE, Laurent (2009): Le traducteur professionnel face aux textes techniques et à la recherche documentaire. Thèse de doctorat non publiée. Paris: ESIT - Université Sorbonne NouvelleParis 3.

LörsCher, Wolfgang (1996): A psycholinguistic analysis of the translation process. Meta. 41(1):26-32.

Mareschal, Geneviève (1989) : Repérage d'unités terminologiques dans le contexte de l'enseignement de la traduction spécialisée. Meta. 34(3):377-380.

Mc Donough, Julie (2007): How do language professionals organize themselves? An overview of translation networks. Meta. 52(4):793-815.

Plassard, Freddie (2007a): Lire pour traduire. Paris: Presses Sorbonne Nouvelle.

Plassard, Freddie (2007b): La traduction face aux nouvelles pratiques en réseaux. Meta. 52(4):643-657.

Tirkkonen-Condit, Sonia (1989): Professional vs. non-professional. In: Candace SÉGuinot, dir. The Translation Process. Toronto: School of Translation - York University, 73-85. 